\title{
Variables implicadas en la adquisición de Competencias Específicas: percepción del futuro Maestro de Educación Primaria
}

\section{Variables involved in the acquisition of specific skills: Perceptions of Primary School Trainees}

\author{
Maria luisa Garcia-Hernandez*1 \\ luisagarcia@um.es \\ Maria Angeles Cano-Muñoz** \\ macano@ucam.edu \\ * Universidad de Murcia, España \\ ** Universidad Católica de Murcia, España
}

\section{Resumen:}

Tomando como punto de referencia el marco Europeo de Educación Superior, este trabajo tiene como objetivo principal conocer desde la percepción del alumnado -aún en proceso de formación- del Grado de Maestro en Educación Primaria, cuáles son las variables que han influido en la adquisición de las competencias específicas que debe adquirir para el desarrollo de su perfil profesional. Para llevar a cabo este trabajo, se ha empleado una metodología no experimental de naturaleza descriptiva, aplicando para la recogida de datos de la muestra $(n=109)$ un cuestionario cerrado ad-hoc. A partir del análisis de las respuestas, se revelan cuáles son las variables más influyentes en la adquisición de las competencias específicas y las que los alumnos universitarios consideran menos desarrolladas para alcanzar dichas competencias. Entre los resultados a des-

\begin{abstract}
:
Taking the European Framework of Higher Education as a point of reference, this article explores the perception of trainees enrolled in the Master's Degree in Primary Education with regard to the variables that have influenced the acquisition of the specific competences that they must acquire for the development of their professional profile. To conduct this study, a non-experimental methodology of a descriptive nature was used, applying a closed ad-hoc questionnaire for the collection of data from the sample $(n=109)$. The analysis of the responses reveals the most influential variables in the acquisition of the specific competences and those variables that university students consider least developed to achieve such competences. The results indicate that students hold the type of evaluation as the least influential variable regarding the acquisition of the specific
\end{abstract}

1 Dirección para correspondencia (correspondence address):

Maria Luisa Garcia- Hernandez. Facultad de Educación, Universidad de Murcia. Calle Campus Universitario, 12, 30100 Murcia (España). E-mail: luisagarcia@um.es 
Variables implicadas en la adquisición de Competencias Específicas: percepción del futuro Maestro de Educación Primaria

Maria Luisa Garcia-Hernandez y Maria Angeles Cano-Muñoz

tacar y según el alumnado, el tipo de evaluación es la variable que menos ha influido en la adquisición de las competencias específicas del título. Con los datos que se muestran a continuación, se pretende realizar un acercamiento a los procesos educativos que tienen lugar en las aulas universitarias desde las percepciones de los alumnos y, sobre todo, analizar su desarrollo competencial como futuros docentes que influirá, de manera inevitable, en su práctica en las aulas escolares.

\section{Palabras clave:}

Enseñanza superior; competencias; docencia universitaria; estudiante universitario; formación. competences of the degree. These data aim to approach educational processes that take place in university classrooms from the perceptions of students and, above all, analyse students' competence development since this will inevitably influence their future teaching.

\section{Key words:}

Higher education; skills; university teaching; university student; academic training.

\section{Résumé:}

En prenant comme point de référence le cadre européen de l'enseignement supérieur, ce travail a comme objectif principal de connaître, à partir de la perception des étudiants -encore en cours de formation- du Diplôme d'Enseignant de I'Enseignement Primaire, quelles sont les variables qui ont influencé l'acquisition des compétences spécifiques qu'ils doivent acquérir pour le développement de leur profil professionnel. Pour réaliser ce travail, on a utilisé une méthodologie non expérimentale de nature descriptive, en appliquant un questionnaire ad hoc fermé pour recueillir les données de l'échantillon $(n=109)$. L'analyse des réponses révèle quelles sont les variables les plus influentes dans l'acquisition des compétences spécifiques et celles que les étudiants universitaires considèrent comme moins développées pour atteindre ces compétences. Parmi les résultats à souligner et selon les étudiants, le type d'évaluation est la variable qui a eu le moins d'influence sur l'acquisition des compétences spécifiques du diplôme. Avec les données présentées ci-dessous, l'objectif est de faire une approche des processus éducatifs qui ont lieu dans les classes universitaires à partir des perceptions des étudiants et, surtout, d'analyser le développement de leurs compétences en tant que futurs enseignants qui influenceront, de manière inévitable, leur pratique dans les classes scolaires.

\section{Mots Clé:}

Enseignement supérieur; compétences; enseignement universitaire; étudiant universitaire; formation.

Fecha de recepción: 16-02-2020

Fecha de aceptación: 09-06-2020 


\section{Introducción}

La Universidad española se halla inmersa desde hace tiempo, sin que esto le reste importancia y vigencia, en el proceso de transición al Espacio Europeo de Educación Superior (EEES), con el referente inmediato del plan Bolonia, configurando una nueva forma de entender la educación (Ramírez, Gutiérrez y Corpas, 2013), que se ha dejado sentir especialmente en el diseño de los planes de estudios y la delimitación de los perfiles académicos y profesionales. La complejidad de este proceso, requiere de reformas permanentes en las acciones formativas llevadas a cabo en la enseñanza superior, empezando por una revisión de las prácticas del profesorado para dar respuesta a las nuevas condiciones en las que se organiza y desarrolla la docencia (Zabalza, 2004).

De este modo, la incorporación de la Universidad española al EEES está ligada a una profunda revisión y transformación en los métodos de enseñanza y evaluación empleados por los docentes, lo que exige cambios tanto en el rol que desempeñan los profesores como los alumnos (García-Merino, Urionabarrenetxea y Bañales-Mallo, 2016; GutiérrezGarcía, Pérez-Pueyo, Pérez-Gutiérrez y Palacios-Picos, 2011). Esto ha conllevado una modificación en la concepción del proceso de enseñanza-aprendizaje, no solamente en cómo es percibido, sino en cómo es aplicado y evaluado. Como indica Zabalza (2004), existe una preocupación en la Universidad por conocer las estrategias a través de las cuales los estudiantes aprenden, ya que únicamente conociendo este proceso, estaremos en condiciones de su modificación o mejora.

El posicionamiento didáctico centrado en la enseñanza del profesor, como fuente principal de información a través de la clásica lección magistral, deja paso a un planteamiento de formación académica en la que el estudiante y su aprendizaje se sitúan en el centro de la institución (Gómez-Puertas, Roca-Cuberes y Guerrero-Solé, 2014; González-Pienda, San Pedro, Rodríguez, Cerezo y González-Castro, 2015). El discente pasa de ser una figura pasiva y dependiente de la enseñanza del profesor, a otra que implica mayor responsabilidad y autonomía en la construcción de su propio aprendizaje, a través de la adquisición de competencias.

En relación con esto último, el aprendizaje por competencias constituye el núcleo del paradigma docente del EEES. Este proceso de convergencia implica una apuesta decidida por las competencias entre sus principales cambios, considerando que un reto de este proceso es la ple- 
na integración de los alumnos universitarios a la vida laboral, a través de la adquisición de competencias relacionadas con su formación integral como personas y profesionales (Arana et al., 2012; Gómez-Puertas et al., 2014). Por estos motivos, la integración efectiva de las competencias en los títulos de Grado es una cuestión que merece la pena ser estudiada con mayor detenimiento (García-San Pedro y Gairín-Sallán, 2011), teniendo en cuenta que el término competencia ha sido ampliamente estudiado en la literatura, pero es difícil de concretar o definir por las posturas contrapuestas que sobre este se ciernen.

Sin detenernos en el concepto, propósito del siguiente apartado, consideramos importante destacar que la formación por competencias aporta transparencia a los perfiles profesionales, por lo que se puede entender como parte de la calidad de la formación y aprendizaje de los estudiantes (García-San Pedro y Gairín-Sallán, 2011). Consecuentemente, la Universidad debe prestar una atención especial a los resultados de aprendizaje de los estudiantes como indicadores de su futuro valor competencial (González y López, 2010; Villa, Campo, Arranz, Villa y García, 2013).

Desde estas consideraciones, parece importante realizar una aproximación a las valoraciones que hacen los propios estudiantes de las competencias adquiridas en su proceso de formación, puesto que serán las que, en un futuro próximo, deban de poner en práctica en su incorporación al mundo laboral. Estas reflexiones Ilevan a la elaboración del estudio que se presenta en este trabajo.

\section{Marco teórico}

A partir de las reflexiones realizadas en el apartado anterior, la base del modelo de formación académica universitaria se encuentra en el aprendizaje por competencias (Gómez-Puertas et al., 2014). Concretamente, nos centraremos en aquellas específicas relacionadas con ámbitos profesionales delimitados. En este sentido, Lenoir y Morales-Gómez (2011) resaltan que el enfoque por competencias viene a transformar profundamente la concepción, objetivos y las modalidades de formación. En otras palabras, cada perfil profesional delimita la adquisición y desarrollo de competencias básicas, ya que "el aprendizaje viene condicionado por todo el conjunto de capacidades y habilidades que poseen los alumnos 
como equipamiento personal y que utilizan adecuadamente como estrategias de uso" (Zabalza, 2004, p. 197). Esta formación no se puede eludir pues, como afirma Rué (2007), el EEES se centra en las competencias que debe adquirir el alumno.

Tras estas afirmaciones, parece lógico plantearnos la siguiente cuestión: ¿qué entendemos por competencias? De acuerdo con la literatura específica, existe una ambigüedad y amplitud semántica asociada a la noción de competencia (Gómez-Puertas et al., 2014), motivado por el hecho de que es uno de los términos habituales de los discursos pedagógicos actuales. Por lo tanto, presentar el sentido de las competencias resulta una tarea compleja,dada la gran disparidad conceptual que procede de los múltiples autores que han escrito a este respecto. A continuación, se presentan algunas de estas aproximaciones conceptuales, haciendo especial alusión a aquellas vinculadas con el ámbito educativo.

En primer lugar, de acuerdo con el proyecto Tuning (2003), el término competencia representa una combinación de atributos (con respecto al conocimiento y sus aplicaciones, aptitudes, destrezas y responsabilidades) que describen el nivel con que una persona es capaz de desempeñarlos (citado en Ramírez et al., 2013). Por su parte, Denyer, Furnémont, Poulain y Vanloubbeeck (2007) indican que las competencias hacen referencia a:

un conjunto de elementos que el sujeto puede movilizar para resolver una situación con éxito hasta aquella que refiere a un conjunto de disposiciones de naturaleza cognitiva, afectiva, reflexiva y contextual. La movilización se expresa por medio del concepto de disposición, para resolver situaciones problema (pp. 36-37).

Otro autor a destacar es Zabalza (2003), quien define competencia como "la capacidad individual para emprender actividades que requieran una planificación, ejecución y control autónomos y la capacidad de usar el conocimiento y las destrezas relacionadas con productos y procesos y, por consiguiente, de actuar eficazmente para alcanzar un objetivo" (p. 31). En definitiva, según este autor la competencia puede ser entendida como la forma en la que los estudiantes se enfrentan a una tarea y las operaciones cognitivas que se ponen en práctica para solventarla (Zabalza, 2004).

Continuando con este repaso de autores, De Miguel (2006) define la 
competencia como una habilidad o capacidad del estudiante para desarrollar una actividad mental. Más concretamente, indica que las competencias expresan la capacidad que tiene un estudiante para afrontar situaciones problemáticas en un determinado contexto académico. El autor destaca que la competencia puede estar supeditada al contexto, condicionada por la situación en la que se debe poner en práctica y puede variar de acuerdo con la experiencia del alumno en dichos campos.

Finalmente, la Agencia Nacional de Evaluación de la Calidad y Acreditación española (2013), conocida por sus siglas como ANECA, especifica que la competencia es "la capacidad de poner en acción conocimiento y habilidades" (p. 6) e incluso "para expresar lo que un estudiante debe saber, entender y ser capaz de hacer al finalizar sus estudios" (p. 21). Por tanto, las competencias específicas hacen referencia a las destrezas que los alumnos, en nuestro caso como futuros profesionales de la educación, deberán haber adquirido.

Estas aproximaciones teóricas vienen a constatar el interés creciente y generalizado por comprender qué son y cómo se adquieren las competencias, pues desde su entendimiento se podrá trabajar mejor para su adquisición y desarrollo en la Universidad. Así pues, teniendo en cuenta las aproximaciones conceptuales presentadas, se puede decir que la competencia no consiste en la adquisición de conocimientos, entendidos como información, tal y como en ocasiones se ha reducido erróneamente el concepto, sino en saber cómo utilizar estos conocimientos de manera útil y eficaz y, para ello, además se requiere de otras disposiciones personales mutuamente relacionadas como capacidades, habilidades y actitudes.

Cabe resaltar, teniendo en cuenta el objeto de estudio de esta investigación,que en la titulación de Magisterio la formación inicial debe dotar a los alumnos de las competencias necesarias para un buen desempeño en su labor profesional, así como garantizar unos procesos de calidad y transcendencia educativa (Romero-Cerezo, 2004). Es por ello que, en el título de Grado de Maestro en Educación Primaria, deberán considerarse las competencias específicas que garanticen la capacitación del alumno para el desempeño de la labor docente (RomeroCerezo, Zagalaz-Sánchez, Romero-Rodríguez y Martínez-López, 2011). La complejidad y diversidad de los procesos educativos, es reflejo de la importancia de la formación en competencias específicas de los futuros maestros. 
En este sentido, debemos detenernos a pensar sobre cómo aprenden los discentes y bajo qué condiciones el aprendizaje mejora, como señala Zabalza(2004). Por tanto, será necesario conocer qué variables influyen en la adquisición de las competencias específicas por parte del alumnado y, por ende, conforman su aprendizaje. Este autor señala aspectos que pueden ser relevantes para el aprendizaje del estudiante, como la práctica, el tipo de trabajo que se le solicite, la atención y la implicación personal que pone en la tarea. Así pues, estas y otras variables pueden influir considerablemente en el proceso de aprendizaje del alumno y, en consecuencia, en la adquisición de las competencias.

En el proceso de enseñanza-aprendizaje confluyen una serie de factores que condicionan este proceso. Para ello, la figura del docente es un aspecto clave del aprendizaje del alumno, como mediador del mismo. Con la implantación del EEES, la figura del docente se ha transformado, adquiriendo como principal función favorecer y guiar al alumno para que pueda acceder intelectualmente a los contenidos teóricos y prácticas profesionales de una determinada disciplina (Pegalajar et al., 2013). Esto implica, como recogen estos autores, una diversificación de la metodología docente, porque las competencias no se pueden alcanzar con una única metodología, tomando un protagonismo especial la introducción de nuevas formas de aprendizaje colaborativo.

Así pues, consideramos que la metodología que desarrolle el docente influirá en el tipo de evaluación y en el tipo de aprendizaje que finalmente alcance el discente, en las actividades que se planteen en la materia, cómo se hacen y qué esfuerzo suponen para el alumno y, por supuesto, en la motivación de este hacia la materia. Estas y otras variables pueden influir en el aprendizaje del estudiante y, por tanto, en la adquisición de unas determinadas competencias.

El objetivo de este estudio es conocer -desde la experiencia del alumnado- la relación entre las competencias específicas del título de Grado de Maestro en Educación Primaria y las variables que influyen en la adquisición de estas competencias específicas, como aspectos clave en el futuro desarrollo profesional del alumnado que, si bien en cualquier formación universitaria es importante, se deja sentir de manera especial en la formación de los futuros formadores, los maestros, para desde la misma impulsar el logro de una educación de calidad en respuesta a las demandas del EEES y las necesidades de la sociedad. 
Variables implicadas en la adquisición de Competencias Específicas: percepción del futuro Maestro de Educación Primaria

Maria luisa Garcia-Hernandez y Maria Angeles Cano-Muñoz

\section{Marco empírico}

Considerando el objetivo que daba fin al apartado anterior, se trata ahora de exponer los siguientes aspectos: metodología, muestra, técnica de recogida de información e instrumento de análisis de datos empleados en esta investigación.

\section{Diseño}

Para acercarnos a las vivencias de los discentes y tomando como referencia las particularidades de este estudio, se consideró oportuno seleccionar una metodología de investigación no experimental de naturaleza descriptiva. Esta investigación es considerada de índole descriptiva puesto que permite identificar las características del fenómeno de estudio, describiendo el estado presente del mismo y sirviendo como base para otras investigaciones (Hernández-Sampieri, Fernández-Collado y Baptista-Lucio, 2010; Salkind, 2009). Puesto que las descripciones se realizan en un momento concreto, también se puede definir como un estudio transversal (León y Montero, 2014).

\section{Muestra}

Para la selección de la muestra, en esta investigación se ha empleado un muestreo por conveniencia, ya que nuestra intención ha sido escoger individuos que cumplan con las características que interesaban partiendo del objetivo del estudio, en este caso, estudiantes del primer curso del Grado en Educación Primaria. Es por ello que centramos nuestro trabajo en el muestreo no probabilístico, pues todos los estudiantes de Magisterio no han tenido la probabilidad igual e independiente de ser seleccionados (Salkind, 2009), sino aquellos que cursaban primero de carrera.

La muestra de estudio estuvo formada por un total de 109 alumnos universitarios, con edades comprendidas entre 18-20 años, que participaron en la investigación de manera voluntaria, habiendo informado previamente del propósito de esta investigación. La mayoría de los alumnos aceptaron participar, resultando una muestra relevante del total de la población de alumnos matriculados en el Grado de Maestro en Educación Primaria (promoción 2015-2019) de la Universidad de Murcia. Finalmente, es importante tener en cuenta, de acuerdo con Bolívar 
(2008), que contar con las propias vivencias (...) que los actores narran se convierte en una perspectiva peculiar de la investigación.

\section{Instrumento y procedimiento}

En cuanto a la técnica de recogida de información señalar que se trata de un cuestionario cerrado elaborado ad-hoc, que fue validado mediante juicio de expertos. Cabe destacar que Utkin (2005, citado en Escobar y Cuervo, 2008) plantea que el juicio de expertos resulta, en muchas áreas, una parte esencial de la información cuando las observaciones experimentales están limitadas. Para la validación de este cuestionario se seleccionaron 3 jueces del ámbito educativo (con experiencia en el ámbito comprendida entre 8 y 10 años), a quienes se les contextualizó y presentó el cuestionario inicial. Tras analizar el instrumento y valorarlo teniendo en cuenta su claridad, adecuación y pertinencia, indicaron una serie de sugerencias para algunos de los ítems del cuestionario con la finalidad de mejorarlo. Evidentemente, estas sugerencias fueron incorporadas, diseñándose de forma definitiva la versión del cuestionario.

Concretamente, en el cuestionario estaba conformado por 12 variables (60 ítems) en los que se le solicitaba al estudiante que seleccionase qué aspectos de los indicados habían influido en la adquisición de cada competencia específica. Así pues, de acuerdo con León y Montero (2004), el cuestionario diseñado recoge una serie de preguntas determinadas previamente, con el objeto de facilitar su posterior codificación, ya que las respuestas solo pueden ser las fijadas en el texto, por tanto, podemos constatar que se trata de un cuestionario de preguntas cerradas. Los estudiantes valoraban de 1-4 (siendo $1=$ muy desacuerdo y $4=$ muy de acuerdo) escala Likert cuál había sido la variable que más había influido en la adquisición de cada competencia. En la Tabla 1, se presentan las competencias y variables que integraban el cuestionario. 
Variables implicadas en la adquisición de Competencias Específicas: percepción del futuro Maestro de Educación Primaria

Maria Luisa Garcia-Hernandez y Maria Angeles Cano-Muñoz

Tabla 1

Cuestionario de recogida de información

Competencias Específicas Grado Primaria

Variables que influyen en la

adquisición de la competencia

CE1. Conocer las áreas curriculares de la Rol del docente

Educación Primaria, la relación interdisci- Metodología

plinar entre ellas, los criterios de evaluación

y el cuerpo de conocimientos didácticos en

torno a los procedimientos de enseñanza y

aprendizaje respectivos.

Evaluación

Motivación

CE2. Diseñar, planificar y evaluar procesos Rol del docente

de enseñanza y aprendizaje, tanto indivi-Metodología

dualmente como en colaboración con otros Evaluación

docentes y profesionales del centro.

Motivación

Actividades y trabajos prácticos

CE3. Abordar con eficacia situaciones de Rol del docente

aprendizaje de lenguas en contextos multi- Metodología

culturales y plurilingües. Fomentar la lectura

y el comentario crítico de textos de los diver-

sos dominios científicos y culturales conteni-

Evaluación

dos en el currículo escolar.

Motivación

CE4. Diseñar y regular espacios de aprendiza- Rol del docente

je en contextos de diversidad y que atiendan Metodología

a la igualdad de género, a la equidad y al res- Evaluación

peto de los derechos humanos que conformen Motivación

los valores de la formación ciudadana.

Actividades y trabajos prácticos

CE5. Fomentar la convivencia en el aula y Rol del docente

fuera de ella, resolver problemas de disci- Metodología

plina y contribuir a la resolución pacífica de conflictos. Estimular y valorar el esfuerzo, la constancia y la disciplina personal en los estudiantes.

Evaluación

Motivación

CE6. Conocer la organización de los colegios de educación primaria y la diversidad de acActividades y trabajos prácticos ciones que comprende su funcionamiento. Desempeñar las funciones de tutoría y de Metodología orientación con los estudiantes y sus familias, atendiendo a las singulares necesidades Evaluación educativas de los estudiantes. Asumir que el ejercicio de la función docente ha de ir per- Motivación feccionándose y adaptándose a los cambios científicos, pedagógicos y sociales a lo largo Actividades y trabajos prácticos de la vida. 
Competencias Específicas Grado Primaria

Variables que influyen en la adquisición de la competencia

CE7. Colaborar con los distintos sectores de Rol del docente la comunidad educativa y del entorno social. Metodología Asumir la dimensión educadora de la función Evaluación docente y fomentar la educación democráti- Motivación ca para una ciudadanía activa. Actividades y trabajos prácticos ma respecto de los saberes, los valores y las Metodología instituciones sociales públicas y privadas. Evaluación Motivación

Actividades y trabajos prácticos

CE9. Valorar la responsabilidad individual Rol del docente y colectiva en la consecución de un futuro Metodología sostenible.

Evaluación

Motivación

Actividades y trabajos prácticos

CE10. Reflexionar sobre las prácticas de aula Rol del docente para innovar y mejorar la labor docente. Adquirir hábitos y destrezas para el aprendizaje autónomo y cooperativo y promoverlo entre los estudiantes. Metodología

Evaluación Motivación Actividades y trabajos prácticos

CE11. Conocer y aplicar en las aulas las tec- Rol del docente nologías de la información y la comunica- Metodología

ción. Discernir selectivamente la información au-
diovisual que contribuya a los aprendizajes, Motivación a la formación cívica y a la riqueza cultural. Actividades y trabajos prácticos CE12. Comprender la función, las posibilida- Rol del docente des y los límites de la educación en la sociedad actual y las competencias fundamentales Metodología que afectan a los colegios de educación pri- Evaluación maria y a sus profesionales. Conocer modelos de mejora de la calidad con aplicación a los centros educativos. Motivación Actividades y trabajos prácticos

Considerando la información presentada en la Tabla 1, lo que se pretendía con el cuestionario aplicado era que los estudiantes, teniendo en cuenta las experiencias vividas a lo largo de su formación como Maestros de Educación Primaria, fuesen capaces de identificar cuál o cuáles 
habían sido las variables que más habían influido en la adquisición de cada competencia específica.

Así pues, considerando la naturaleza del estudio y las características de los datos recogidos, se optó por el tratamiento de la información a través de un análisis cuantitativo, empleando para ello el programa IBM SPSS Statistics 21. Se establecieron las distintas variables y se categorizaron en el programa de análisis, para posteriormente extraer un análisis descriptivo buscando el grado de acuerdo y desacuerdo en las valoraciones que hacían los estudiantes de cada ítem, cuyos resultados más relevantes se exponen y analizaremos de manera pormenorizada en el siguiente apartado.

\section{Resultados y Discusión}

Hasta el momento se ha realizado un recorrido por los aspectos más destacados. Finalmente, en este apartado se analizan y exponen los datos más destacados de este estudio. Como podemos observar en la Figura 1 , se presentan los porcentajes asignados a cada una de las variables en función de cada una de las doce competencias específicas del Grado de Maestro en Educación Primaria.

En este sentido, destacar que cada una de las variables que pueden influir en la adquisición de las competencias tiene asignado una tonalidad diferente con la finalidad de facilitar la comprensión de la imagen. Por otro lado, indicar que seguidamente se analizarán los datos siguiendo el orden en el que se presentan las competencias para ayudar a su presentación e interpretación.

Atendiendo a los datos que aparecen en la gráfica posterior, podemos comprobar que en el eje de ordenadas se recogen las doce competencias específicas, mientras que en el eje de abscisas aparecen las diferentes variables que han contribuido,en mayor o menor medida, a la consecución de cada una de las competencias.

Comenzaremos analizando la competencia CE1: "Conocer las áreas curriculares de la Educación Primaria, la relación interdisciplinar entre ellas, los criterios de evaluación y el cuerpo de conocimientos didácticos en torno a los procedimientos de enseñanza y aprendizaje respectivos". Teniendo en cuenta los datos la siguiente figura, podemos constatar que -de acuerdo con la experiencia de los estudiantes universitarios la 


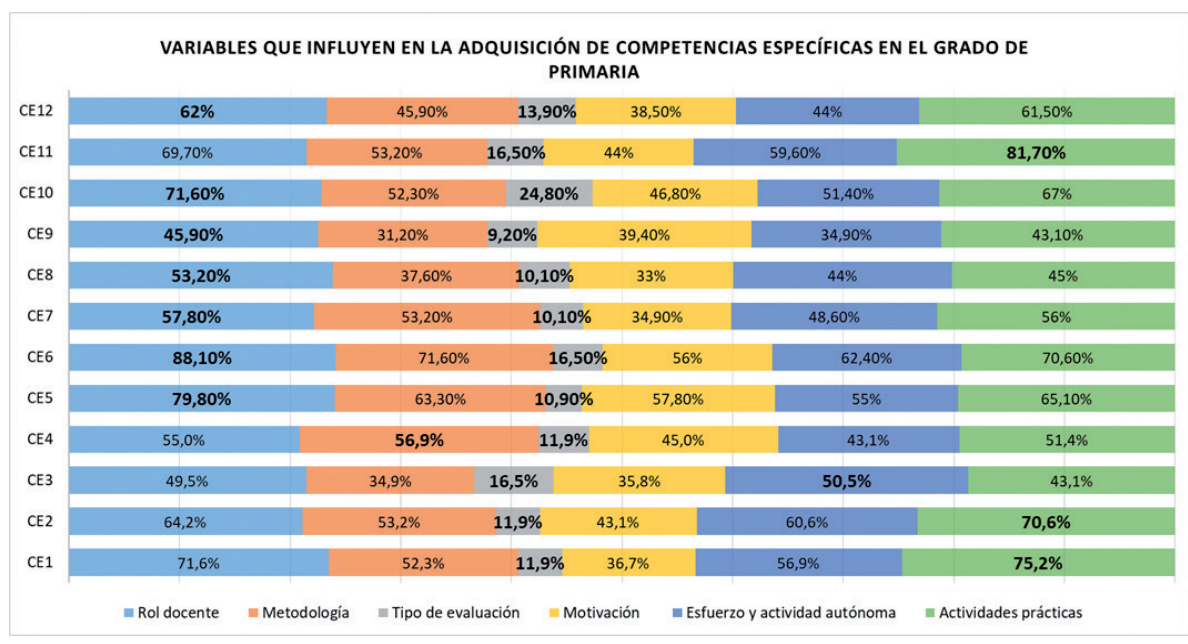

Figura 1. Variables que influyen en la adquisición de las competencias específicas en el Grado de Educación Primaria.

variable más influyente en la adquisición de esta CE1 han sido las actividades prácticas $(75,2 \%)$. Otra de las variables que ha favorecido esta competencia han sido: el rol docente $(71,6 \%)$, el esfuerzo y la actividad autónoma $(56,9 \%)$ y la metodología $(52,3 \%)$ son factores que intervienen de manera determinante en el desarrollo de esta CE. Mientras que, por el contrario, resaltan que el tipo de evaluación $(11,9 \%)$ y la motivación $(36,7 \%)$ son las variables que menos han favorecido la adquisición de esta primera competencia.

En cuanto a la CE2 "Diseñar, planificar y evaluar procesos de enseñanza y aprendizaje, tanto individualmente como en colaboración con otros docentes y profesionales del centro" los alumnos manifiestan con sus respuestas que las variables que más han favorecido la adquisición de esta competencia han sido: las actividades prácticas (70,6\%), el rol docente $(64,2 \%)$, el esfuerzo y la actividad autónoma y la metodología $(53,2 \%)$, mientras que el tipo de evaluación $(11,9 \%)$ y la motivación $(43,1 \%)$, como sucedía con la CE1, no han influido de manera determinante en la habilidad para aplicar esta competencia.

Por otra parte, han valorado que la CE3, enfocada a "abordar con eficacia situaciones de aprendizaje de lenguas en contextos multiculturales y plurilingües. Fomentar la lectura y el comentario crítico de textos de los diversos dominios científicos y culturales contenidos en el currículo 
escolar", ha sido desarrollada a través de variables como el esfuerzo y la actividad autónoma (50,5\%), rol docente (49,5\%) y las actividades prácticas $(43,1 \%)$. Sin embargo, resulta llamativo que los alumnos consideren que ninguna de las otras variables ha influido de manera determinante en la adquisición de esta competencia. En este sentido, identifican que: el tipo de evaluación (16,5\%); la metodología $(34,9 \%)$ y la motivación $(35,8 \%)$ han sido variables poco determinantes en la puesta en práctica de esta competencia.

Respecto a la CE4 "Diseñar y regular espacios de aprendizaje en contextos de diversidad y que atiendan a la igualdad de género, a la equidad y al respeto de los derechos humanos que conformen los valores de la formación ciudadana" los alumnos consideran que las variables más influyentes han sido: la metodología empleada (56,9\%), el rol docente $(55 \%)$ y las actividades prácticas $(51,4 \%)$, la motivación $(45 \%)$ y el esfuerzo y la actividad autónoma (43,1\%). Sin embargo, coinciden en destacar que los aspectos que menos han influido en el desarrollo de esta competencia ha sido el tipo de evaluación (11,9\%),

Tal y como se puede inferir de los análisis realizados hasta el momento, tomando como referencia las cuatro primeras competencias específicas, se distinguen unos patrones en las valoraciones de los alumnos encuestados, en los que dejan entrever cuáles han sido las variables que más condicionan la adquisición de competencias y aquellas que influyen de manera más circunstancial. No obstante, continuamos con el análisis de las siguientes competencias de CE5.

En cuanto a la Competencia Específica 5 "Fomentar la convivencia en el aula y fuera de ella, resolver problemas de disciplina y contribuir a la resolución pacífica de conflictos. Estimular y valorar el esfuerzo, la constancia y la disciplina personal en los estudiantes", destacar que para su desarrollo han sido imprescindibles según los alumnos variables como: el rol docente $(79,80 \%)$, las actividades prácticas $(65,10 \%)$, la metodología $(63,30 \%)$, la motivación $(57,80 \%)$ y el esfuerzo y la actividad autónoma (55\%); mientras que el tipo de evaluación (10,9\%) no ha influido de manera determinante en la adquisición de esta competencia.

Por otro lado, en cuanto a la CE6 dedicada a "Conocer la organización de los colegios de educación primaria y la diversidad de acciones que comprende su funcionamiento. Desempeñar las funciones de tutoría y de orientación con los estudiantes y sus familias, atendiendo a las singulares necesidades educativas de los estudiantes. Asumir que el ejer- 
cicio de la función docente ha de ir perfeccionándose y adaptándose a los cambios científicos, pedagógicos y sociales a lo largo de la vida", los alumnos valoran que: el rol docente $(88,10 \%)$, la metodología $(71,60 \%)$, las actividades prácticas $(70,60 \%)$, el esfuerzo y la actividad autónoma $(62,40 \%)$ y la motivación $(56 \%)$ han sido esenciales para alcanzar y desarrollar de manera adecuada esta competencia. Sin embargo, la variable que menos ha influido ha sido el tipo de evaluación (16,50\%).

Respecto a la CE7 orientada a "Colaborar con los distintos sectores de la comunidad educativa y del entorno social. Asumir la dimensión educadora de la función docente y fomentar la educación democrática para una ciudadanía activa" podemos constatar que según las vivencias de los alumnos: el rol docente $(57,80 \%)$, las actividades prácticas $(56 \%)$, la metodología $(53,20 \%)$ y el esfuerzo y actividad autónoma $(48,6 \%)$ han influido de manera determinante para adquirir esta; por el contrario, señalan que el tipo de evaluación (10,10\%) y la motivación (34,9\%) no han sido variables determinantes para la consecución de esta competencia.

Respecto a la CE8 orientada a "Mantener una relación crítica y autónoma respecto de los saberes, los valores y las instituciones sociales públicas y privadas" los alumnos reconocen que las variables que ha influido en el desarrollo de la misma ha sido el rol docente $(53,20 \%)$, las actividades prácticas (45\%), esfuerzo y actividad autónoma (44\%),mientras que el resto de variables (tipo de evaluación (10,10\%), motivación $(33 \%)$ y metodología $(37,6 \%)$, no han influido de manera determinante para desarrollar dicha competencia.

Del mismo modo, sobre la CE9 destinada a "Valorar la responsabilidad individual y colectiva en la consecución de un futuro sostenible" destacar que, según la experiencia de los alumnos, no les ha influido de manera significativa ninguna de las variables expuestas en este estudio. No obstante, las más valoradas por los estudiantes han sido rol docente $(45,9 \%)$, y actividades prácticas $(43,10 \%)$. Por este motivo sería interesante, para futuras investigaciones, ampliar el rango de variables por si consideran que han sido otros aspectos decisivos en el desarrollo de este saber. No obstante, es un dato relevante que hemos querido hacer constar.

En cuanto a la CE10 "Reflexionar sobre las prácticas de aula para innovar y mejorar la labor docente. Adquirir hábitos y destrezas para el aprendizaje autónomo y cooperativo y promoverlo entre los estudian- 
tes", las variables más destacadas para el fomento de esta competencia específica han sido: el rol docente $(71,60 \%)$, las actividades prácticas $(67 \%)$, la metodología (52,30\%); el esfuerzo y la actividad autónoma $(51,40 \%)$ y la motivación (46,8\%); mientras que el tipo de evaluación $(24,8 \%)$ no ha sido para el desarrollo de esta competencia.

La CE11 orientada a "Conocer y aplicar en las aulas las tecnologías de la información y la comunicación. Discernir selectivamente la información audiovisual que contribuya a los aprendizajes, a la formación cívica y a la riqueza cultural" ha sido fomentada a través de variables como: las actividades prácticas $(81,70 \%)$, el rol docente $(69,70 \%)$, el esfuerzo y la actividad autónoma $(59,60 \%)$, la metodología $(53,20 \%)$ y la motivación (44\%); mientras que la evaluación (16,5\%) no ha sido determinante para la adquisición de la misma según los alumnos encuestados.

Finalmente, la CE12 relacionada con "Comprender la función, las posibilidades y los límites de la educación en la sociedad actual y las competencias fundamentales que afectan a los colegios de educación primaria y a sus profesionales. Conocer modelos de mejora de la calidad con aplicación a los centros educativos" los estudiantes manifiestan que el rol docente $(62 \%)$ y las actividades prácticas $(61,50 \%)$ han sido las dos variables más influyentes en la adquisición de esta capacidad; seguidas de la metodología (45,9\%) y el esfuerzo y actividades prácticas (44\%). Mientras que el tipo de evaluación (13,9\%) no ha sido determinante.

A partir del análisis descriptivo presentado sobre los principales resultados obtenidos en esta investigación, que se desprenden de la Figura 1, cabe destacar que existen unas tendencias claras en las valoraciones de los estudiantes, vinculadas con aquellas variables que, según su percepción, han sido determinantes en el desarrollo de dichas competencias. Del mismo modo, los discentes constatan que hay variables que, pese a su importancia (como puede ser la evaluación), no han sido influyentes o no han contribuido de manera relevante en la adquisición de las competencias de la carrera. En el siguiente apartado de conclusiones, se señalan algunos de los aspectos más destacados de esta investigación.

\section{Conclusiones}

En el trabajo presentado, tal y como recogen De la Mano-González y Moro-Cabero (2009), una de las primeras cuestiones a las que tratamos 
de dar respuesta se relaciona con qué competencias deben ser adquiridas en una determinada titulación, concretamente en esta investigación en el Grado de Maestro en Educación Primaria, identificando además qué variables han favorecido la adquisición de estas competencias pues, desde el conocimiento de las mismas, se pueden hacer propuestas para orientar el desarrollo y adquisición de las competencias básicas para el ejercicio profesional.

Así pues, teniendo en cuenta el análisis realizado sobre las doce competencias específicas, se puede comprobar que existen una serie de variables que tienden a mantenerse como las más valoradas por los alumnos para la adquisición de estas competencias del título, mientras que existen unos patrones en la valoración de otras variables consideradas poco influyentes en su desarrollo durante la carrera.

Una de las conclusiones más relevantes a las que hemos llegado tras este estudio es que el tipo de evaluación (teniendo en cuenta la percepción de los estudiantes que son los que han trabajado para adquirir estas competencias, de ahí la importancia de su percepción) ha sido la variable que, según sus vivencias, menos ha influido en la adquisición de las competencias específicas en general. Sin embargo, pese a lo que se puede pensar en un primer momento y teniendo en cuenta la literatura especializada, la evaluación es uno de los elementos clave del proceso de enseñanza-aprendizaje, especialmente por el volumen de información que facilita al profesor (Rodríguez-López, 2002). Además, es importante tener en cuenta que la evaluación tiene una función reguladora del aprendizaje, puesto que las decisiones sobre cómo estudiar la materia están condicionadas por el tipo de evaluación al que se enfrentan los alumnos (Murphy, 2006; Pérez-Cabaní y Carretero-Torres, 2003; Villardón-Gallego, 2006).

Otro aspecto a destacar tras este estudio ha sido que la variable motivación no ha influido, en la mayoría de ocasiones, en la adquisición de las competencias específicas de acuerdo con las vivencias de los alumnos del Grado en Educación Primaria. Así, Ilama la atención que esta variable de naturaleza propiamente intrínseca del alumnado no sea considerada de gran relevancia para la adquisición de las competencias básicas de la carrera.

Por otra parte, en algunas competencias los estudiantes señalan que no les han influido las variables indicadas en este estudio para el desarrollo de las competencias específicas. Más concretamente, señalan que 
en la competencia 9 (referente a valorar la responsabilidad individual y colectiva en la consecución de un futuro sostenible) no les ha influido ninguna, mientras que en la competencia 3 (que alude a abordar con eficacia situaciones de aprendizaje en contextos multiculturales y fomentar la lectura y comentario de textos de los diversos dominios científicos) y 8 (referida a mantener una relación crítica y autónoma respecto a los saberes y valores de diferentes instituciones) únicamente señalan la influencia de una sola variable.

Asimismo, resaltar que los alumnos coinciden en constatar que hay variables que son fundamentales para la adquisición de las competencias profesionalizadoras, como son el rol docente, la metodología y las actividades prácticas. En este sentido, se puede resaltar que el EEES ha mejorado el grado de satisfacción de los alumnos respecto al profesorado y la aplicación de los conocimientos, de acuerdo con Otero, Ferro y Vila (2012, citado en Pegalajar et al., 2013). En concreto, en lo que respecta al rol docente, podemos confirmar que para asegurar una docencia de calidad en consonancia con los retos que se demandan a la Universidad, es necesario definir un perfil transferencial, flexible y polivalente de este profesional, para que sea capaz de adecuarse a la diversidad y a los continuos cambios que se vienen dando en la sociedad actual (Bozu y Canto-Herrera, 2009).

Como síntesis final, remarcar que este estudio ha dado luz a los objetivos y cuestiones que a su inicio nos planteamos. De este modo, hemos podido conocer cuáles son las variables más influyentes según las vivencias de los alumnos para el desarrollo de las competencias específicas en el Grado en Educación Primaria y aquellas que consideran que no han sido significativas en su aprendizaje. Al mismo tiempo, se han abierto otros interrogantes a través del estudio, como futuras líneas de investigación, sobre los que ya se están trabajando, como por ejemplo qué capacidades cognitivas -recuperación, comprensión, análisis y aplicación del conocimiento, tomando como referencia la taxonomía de Marzano y Kendall (2007)- aplican los alumnos para cada competencia específica. Esta última cuestión es complementaria al texto presentado para no sólo comprender la práctica que se desarrolla en las aulas universitarias, sino para valorar mejor cómo esta conforma el perfil de los discentes que en un próximo futuro serán docentes, considerados pilares fundamentales, como decíamos al inicio de este trabajo, para alcanzar mayores cuotas de calidad en la educación, de acuerdo a las exigencias del EEES. 
Variables implicadas en la adquisición de Competencias Específicas: percepción del futuro Maestro de Educación Primaria

Maria luisa Garcia-Hernandez y Maria Angeles Cano-Muñoz

\section{Referencias}

ANECA (2005). Libro Blanco. Título de Grado en Pedagogía y Educación Social. Recuperado de http://www.aneca.es/var/media/150392/libroblanco_pedagogia1_0305.pdf

ANECA (2013). Informe de Resultados. Recuperado de http://www.aneca.es/content/ download/12765/158329/file/learningoutcomes_v02.pdf

Arana, J. M., Ortiz V., Jenaro, C., García, J. J., Zubiauz, B. y Mayor, M. A. (2012). Evaluación de la adquisición de competencias transversales en una asignatura de primero de Grado de Psicología. En M. T. Tortosa, J. D. Álvarez y N. Pellín (Eds.), X Jornadas de Redes de Investigación en Docencia Universitaria (pp. 1838-1847). Alicante: Instituto de Ciencias de la Educación.

Ballester, A. (2002). El aprendizaje significativo en la práctica. Palma de Mallorca: Cibereduca, Recuperado de http://www.aprendizajesignificativo.es/mats/El_aprendizaje_significativo_en_la_practica.pdf

Bolívar, A. (2008). El discurso de las competencias en España: educación básica y educación superior. Red U. Revista de Docencia Universitaria, 6(2), 1-23. doi:10.4995/ redu. 2008.6268

Bozu, Z. y Canto-Herrera, P. J. (2009). El profesorado universitario en la sociedad del conocimiento: competencias profesionales docentes. Revista de Formación e Innovación Educativa Universitaria, 2(2), 87-97.

De la Mano-González, M. y Moro-Cabero, M. (2009). La evaluación por competencias: propuesta de un sistema de medida para el grado de Información y Documentación. BiD: textos universitaris de biblioteconomía i documentació,23,1-16.

De Miguel, M. (2006). Metodologías de enseñanza y aprendizaje para el desarrollo de competencias. Madrid: Alianza.

Denyer, M., Furnémont, J., Poulain, R. y Vanloubbeeck, G. (2007). Las competencias en la educación. Un balance. México: FCE.

Fernández March, A. (2006). Metodologías activas para la formación de competencias. Educatio Siglo XXI, 24, 35-56.

García-Merino, J. D., Urionabarrenetxea, S. yBañales-Mallo, A. (2016). Cambios en metodologías docentes y de evaluación: ¿Mejoran el rendimiento del alumnado universitario? Redie. Revista Electrónica de Investigación Educativa, 18(3), 1-18.

García-San Pedro, M. J. y Gairín-Sallán, J. (2011). Los mapas de competencias: una herramienta para mejorar la calidad de la formación universitaria. REICE. Revista Iberoamericana sobre Calidad, Eficacia y Cambio en Educación, 9(1), 85-102.

Gómez-Puertas, L., Roca-Cuberes, C. y Guerrero-Solé, F. (2014). ¿Cómo perciben los estudiantes la adquisición de competencias? Análisis comparado: Teorías de la comunicación en la Universidad PompeuFabra. Revista Historia y Comunicación Social, 19, 313-326. doi: 10.5209/rev_HICS.2014.v19.45030

González, I. y López, A B. (2010). Sentando las bases para la construcción de un modelo de evaluación a las competencias docentes del profesorado universitario. RIE: Revista de Investigación Educativa, 28(2), 403-423.

González-Pienda, J., San Pedro, J., Rodríguez, C., Cerezo, R. y González-Castro, M. (2015). La formación del profesorado de Educación Infantil y Primaria en el mar- 
Variables implicadas en la adquisición de Competencias Específicas: percepción del futuro Maestro de Educación Primaria

Maria luisa Garcia-Hernandez y Maria Angeles Cano-Muñoz

co del EEES. Revista de estudios e investigación en psicología y educación, 2(1), 1-10.

González, J. y Wagenaar, R. (2003).Tuning Educational Structures in Europe. Bilbao: Universidad de Deusto.

Gutiérrez-Tapias, M., García-Cué, J. L. y Vieira Barros, D. M. (2012). Estudio de las variables que influyen en los estilos de aprendizaje de diferentes grupos de alumnos del Grado de Magisterio de la Universidad de Valladolid, España. Revista estilos de aprendizaje, 10(10), 1-14.

Gutiérrez-García, C., Pérez-Pueyo, A., Pérez- Gutiérrez, M. y Palacios-Picos (2011). Percepciones de profesores y alumnos sobre la enseñanza, evaluación y desarrollo de competencias en estudios universitarios de formación de profesorado. Cultura y educación, 23(4), 499-514.

Hernández-Sampieri, R., Fernández-Collado, C. y Baptista-Lucio, P. (2010). Metodología de la investigación. México: McGraw Hill.

Lenoir, Y. y Morales-Gómez, M. A. (2011). El enfoque por competencias y profesionalización de la enseñanza: una clarificación conceptual. REICE. Revista Iberoamericana sobre Calidad, Eficacia y Cambio en Educación, 9(1), 46-64.

León, O. y Montero, I. (2004). Métodos de investigación en Psicología y Educación. Madrid: McGraw-Hill.

Marzano, R. J. y Kendall, J. S. (2007). The New Taxonomy of Educational Objectives. ThousandOaks, CA: Corwin Press.

Montero Mesa, L. (2018). Relaciones entre teoría y práctica en la formación inicial. Percepciones de formadores y estudiantes del Grado de Maestro en Educación primaria. Educatio Siglo XXI, 36(2), 303-330.

Murphy, R. (2006). Evaluating new priorities for assessment in higher education. En C. Bryan y K. Clegg (eds.), Innovative Assessment in Higher Education (pp. 37-47).New York: Routledge.

Pegalajar, M. C., Pérez, E. y Colmenero, M. J. (2013). Valoración del Espacio Europeo de Educación Superior, según el nivel educativo del alumnado universitario. Innovación Educativa, 13(61), 67-84.

Pérez-Cabaní, M. L. y Carretero-Torres, M. R. (2003). La promoción de estudiantes estratégicos a través del proceso de evaluación que proponen los profesores universitarios. En C. Monereo y J. I. Pozo (Coords.), La Universidad ante la nueva cultura educativa (pp. 173-190). Madrid: Síntesis.

Perrenoud, P. (2005). Diez nuevas competencias para enseñar. Educatio Siglo XXI, 23, 223-229.

Ramírez-García, A., Gutiérrez-Arenas, M. P. y Corpas-Reina, C. (2013). Valoración por parte del alumnado del Grado de Maestro en Educación Infantil de las competencias que se les exige para convertirse en Profesionales de la Educación. En E. Nieto, A. I. Callejas y O. Jerez (Coords.), Las competencias básicas. Competencias profesionales del docente (pp. 179-186). Ciudad Real: Universidad de Castilla-La Mancha.

Rodríguez-López, J. M. (2002). La evaluación en la Universidad: la evaluación del aprendizaje de los alumnos universitarios. En C. Mayor (Coord.), Enseñanza y aprendizaje en la Educación Superior (pp. 161-180). Barcelona: Octaedro. 
Variables implicadas en la adquisición de Competencias Específicas: percepción del futuro Maestro de Educación Primaria

Maria luisa Garcia-Hernandez y Maria Angeles Cano-Muñoz

Romero-Cerezo, C. (2004). Argumentos sobre la formación inicial de los docentes en educación física. Profesorado. Revista de currículum y formación del profesorado, $8(1), 1-20$.

Romero-Cerezo, C., Zagalaz-Sánchez, M. L., Romero Rodríguez, M. N. y Martínez-López, E. J. (2011). Importancia de las competencias profesionales de los Maestros en Educación Física expresadas por los estudiantes. Revista Retos. Nuevas tendencias en Educación Física, Deporte y Recreación, 19, 62-68.

Salkind, N. (2009). Exploring Research. New Jersey: Pearson Education.

Universidad de Murcia (2010). Título de Grado en Pedagogía. Recuperado de https:// www.um.es/c/document_library/get_file?uuid=55980f18-4f59-48ca-b4191a152105ddc3 \&groupld=299436

Villa, A., Campo, L., Arranz, S., Villa, O. y García, A. (2013). Valoración del profesorado de Magisterio sobre el aprendizaje de competencias implantado. Profesorado. Revista de currículum y formación del profesorado, 17(3), 35-55.

Villardón-Gallego, M. L. (2006). Evaluación del aprendizaje para promover el desarrollo de competencias. Educatio Siglo XXI, 24, 57-76.

Yániz, C. (2008). Las competencias en el currículo universitario: implicaciones para diseñar el aprendizaje y para la formación del profesorado. RedU. Revista de Docencia Universitaria, 1, 1-14. doi: 10.4995/redu.2008.6281

Zabalza, M. A. (2003). Competencias docentes del profesorado universitario: Calidad del desarrollo profesional. Madrid: Narcea.

Zabalza, M. A. (2004). La enseñanza universitaria. El escenario y sus protagonistas. Madrid: Narcea. 
